\title{
Necrotizing enterocolitis in a full term infant
}

\author{
Maria Catalina Vaz Ferreira*, Mario Moraes \\ Department of Neonatology, Pereira Rossell Children's Hospital, Montevideo, Uruguay
}

\begin{abstract}
This report details the case of a full-term infant, who developed Necrotizing Enterocolitis (NEC) during the first 24 hours of life. While most cases of NEC occur among patients already admitted to the NICU for a variety of conditions, this case develops in a previously healthy newborn who developed early-onset disease. Our analysis describes the factors predisposing to this condition and discusses the most effective antimicrobial treatment strategies. The focus of the article is to highlight the role of certain risk factors (alterations in the gut microbiome, hypertonic formula feeds, perinatal hypoxia and patent ductus arteriosus with left-to-right shunt) in tipping the scale towards the development of NEC.

Keywords: necrotizing enterocolitis, full-term, microbiome, breastfeeding
\end{abstract}

\section{Introduction}

Necrotizing enterocolitis (NEC) is often a devastating condition primarily affecting preterm infants and is the most common surgical emergency in that population. Overall mortality for NEC ranges from 20 to $40 \%$ [14]. Ninety percent of the affected infants are born preterm, with an age of onset inversely related to gestational age at birth. Approximately 7 to $10 \%$ of all NEC cases occur in babies born after 37 weeks' gestation [1], nevertheless, in our unit no NEC cases in term neonates have been reported in the past three years. The onset of the disease occurs earlier in full term than in preterm infants, and this is possibly due to the pathogenic mechanism [5]. Term babies who develop NEC after 7 days of life (late onset) show a higher mortality [6]. In this population, the

Received: September 2016; Accepted after review: November 2016; Published: December 2016.

${ }^{*}$ Corresponding author: Maria Catalina Vaz Ferreira, MD, Department of Neonatology, Pereira Rossell Children's Hospital, University of the Republic, Montevideo, Uruguay

Email: catalinavazferreira@gmail.com pathophysiology differs from that of premature infants, and is commonly associated with other risk factors such as congenital heart disease, asphyxia, intrauterine growth restriction, polycythaemia, sepsis, or any other cause of deficient mesenteric perfusion. Many controversies currently surround the underlying causes of NEC. However, recent studies have provided new insights on the pathogenic mechanism, suggesting additional contributing factors for the development of the condition. It seems to be clear that NEC in term infants is a multifactorial entity in which predisposing events such as mesenteric hypoperfusion and hypoxia-ischemia are a necessary but insufficient condition.

\section{Case report}

An infant male was born via vaginal delivery at 41 weeks to a 34-year old G1P0 mother who had been treated with a 3-day course of antibiotics for a skin infection. She was an occasional marijuana user. The pregnancy was well controlled; complicated only by a urinary tract infection which was adequately treated. The mother was found to be HIV and Group B Streptococcus negative. 
Labour was induced over three days with oxytocin. Membranes ruptured spontaneously 12 hours prior to delivery and showed clear amniotic fluid. Delivery took place after a prolonged expulsion stage.

The infant was born crying. APGAR scores were 9 and 10 at 1 and 5 minutes respectively. Birth weight was $3340 \mathrm{~g}$. No cord gas was taken at that time. The initial exam was normal and did not reveal any phenotypic alterations. The baby was subsequently sent with his mother. During his first hours of life he was fed breast milk and formula q3 hours and had one stool. Ten hours after birth, the infant was hyporesponsive, had poor suck and one bilious emesis. The physical exam found (change found to revealed) generalized hypotonia, poor perfusion, pale skin, tachypnea and respiratory grunting. The abdomen was distended and tense on gentle palpation, but no masses were identified. Bowel sounds were present. Preductal saturations were above $95 \%$, temperature $36,2 \mathrm{C}$, glucose $94 \mathrm{mg} / \mathrm{dl}$ and BP $76 / 50 \mathrm{mmHg}$, MAP $58 \mathrm{mmHg}$. The infant was admitted to the NICU with the presumptive diagnosis of perinatal infection, and was started empirically on antibiotic treatment with AmpicillinGentamicin.

A few hours later the infant's clinical status deteriorated as evidenced by increased work of breathing along with significant abdominal distension and a shiny bluish abdominal discoloration. Blood was detected in the stools, and the infant was hemodynamically unstable and oliguric (1 $\mathrm{ml} / \mathrm{kg} / \mathrm{hr})$. Consequently, he was placed on mechanical ventilation and umbilical catheters were inserted. Inotropic support and Metronidazole were initiated at that point.

Laboratory tests:

- Blood gas: pH: 7.37; $\mathrm{PCO}_{2}: 22.3 \mathrm{mmHg}$ $\mathrm{PO}_{2}: 137 \mathrm{mmHg} ; \mathrm{HCO}_{3}: 16,1 \mathrm{mmol} / \mathrm{L} ; \mathrm{BE}:-$ $12.7 \mathrm{mmol} / \mathrm{L}$; Lactate: $7,4 \mathrm{mmol} / \mathrm{L}$; Glucose: $179 \mathrm{mg} / \mathrm{dL}$.

- CBC: Hb: 18,1g/dL; Hct: 59\%; WBC: 23.400/ $\mathrm{mm}^{3}$; Plt: 208000/ $\mathrm{mm}^{3}$; RCP:32 (+); PCT:13,1 (-).

- Coagulation: APTT: 56sec; PT: $36 \%$ Fibrinogen: $225 \mathrm{mg} / \mathrm{dL}$.

- Echocardiography: Severe pulmonary hypertension, $2 \mathrm{~mm}$ patent ductus arteriosus
(PDA) with bidirectional shunt. Follow up echocardiography at 72 hours of life: $2.5 \mathrm{~mm}$ PDA with significant left to right shunt.

- Chest- abdomen radiography: (Figure 1).

- Abdomen ultrasound: portal venous gas. Mesenteric and celiac vessels Doppler were within normal ranges.

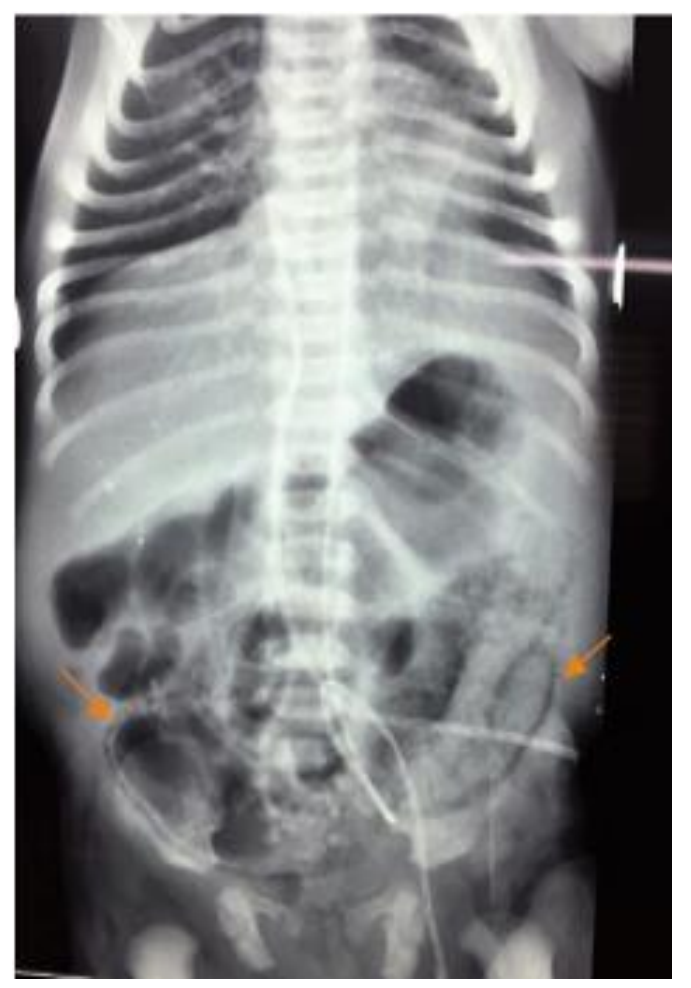

Fig. 1. Extensive pneumatosis, railroad tracks-like (arrows) and dilated bowel loops. No signs of pneumoperitoneum.

Given the presence of hemodynamic instability, initial management consisted of primary peritoneal drainage. The infant developed thrombocytopenia and anemia and required multiple transfusions of platelets and RBC. The baby was clinically stable by 60 hours of life and underwent laparotomy. Findings were almost total colonic necrosis, from rectum to the ascending colon. Subtotal colectomy was performed, including Brooke ascending colostomy with preservation of ileocolic valve and $2 \mathrm{~cm}$ of ascending colon.

Blood and peritoneal cultures were negative, and the infant completed a 10-day course of Ampicillin-Gentamicin and Metronidazole. Trophic feeds were started at seven days of life with expressed breast milk. The ductus closed spontaneously and the 
infant was discharged from the NICU at two weeks of life.

Pathology showed extensive necrosis of the entire colonic wall, mixed inflammatory cell infiltrate, pneumatosis and bacterial colonies in the bowel lumen, consistent with the diagnosis of necrotizing enterocolitis.

\section{Discussions}

NEC in term infants is a rare condition often occurring in infants already admitted to the NICU for another cause. However, this report describes the case of an infant who was initially considered healthy and had no apparent risk factors but developed NEC symptoms shortly after. This leads us to think that there could have been intrauterine factors that contributed to the development of the disease coupled with perinatal events which triggered its onset.

Evidence from several recent studies shows a narrow correlation between genetics, microbiome and nutrition and reveals that the pathogenesis of NEC is mediated via complex interactions between genetic and environmental factors. Evidence further suggests that there is a genetic basis which predisposes the neonate to this devastating condition, and therefore some infants are genetically programmed to be more vulnerable than others [4]. The study of the human genome and genetic polymorphisms (chromosomal microarray), crucial for the development of personalized medicine may also be key to understanding the subtle underlying genetic anomalies that could cause NEC even in the absence of phenotypic alterations [4, 7].

The fact that the mother had been undergoing a course of antibiotics prior to the delivery should be taken into consideration as a possible cause of dysbiosis. Any disturbance of the fragile ecosystem of the infant's microbiome could potentially lead to NEC. The establishment of neonatal gut microbiome, defined as the sum of all microbial life in or on the human gut, begins in utero and it emerges as a result of the vertical transmission of maternal microbiota [8]. Consequently, the infant's microbiota could have been significantly altered by his mother's course of antibiotics. Recent studies suggest the presence of a microbiome within the placenta and meconium, implying that the colonization process begins antenatally and before breastfeeding $[8,9]$, contrary to the previous belief that the in-utero environment was sterile, and that the fetus was first colonized with bacteria at the time of birth. It is now known that there is a direct transfer of maternal intestinal bacteria to the fetus via dendritic cells or other immune cells, maternal mammary glands, breast milk and placental tissue [9].

Another factor that has a profound influence on development of gut microbiome is the infant's diet. The neonate presented in this case had been fed breastmilk, but given that his mother had poor milk production breastfeeding was supplemented with formula. It is well known that breast milk plays a direct role in imprinting the neonatal biome by adding certain bacterial species such as Staphylococcus, Streptococcus, Bifidobacterium and Lactobacillus to the infant's gastrointestinal tract (enteromammary pathway) [8]. Formulafed babies have a different microbial status than those fed human milk [9]. Additionally, breast milk contains immunoglobulins, growth factors, hormones, lactoferrin, glutamine, polyunsaturated fatty acids and oligosaccharides, which give beneficial microbes a specific substrate for growth. This means that by exclusively breastfeeding, the infant may be receiving the probiotics already present in his own mothers' milk. Some studies suggest that babies with reduced mesenteric perfusion who have been fed large volumes of cow's milk based formula are at higher risk of developing NEC [10].

Many of the microbiome's functions are known to be crucial when it comes to protecting the host against pathogens. Intestinal microbiome can limit nutrient resources available to pathogens and foster the development of the barrier's function as it interacts with the innate and adaptive immune systems to establish gut motility and protection against pathogens.

The disruption of the normal intestinal flora (dysbiosis) leads to a proinflammatory state, allowing translocation of pathogens across the 
intestinal epithelia and therefore promoting the development of NEC. Furthermore, colonization with Lactobacillus and bacterial diversity is affected by antibiotic usage, with blooms of Staphylococcus and Enterobacter. This leads to an alteration in the proportion of the commensal bacteria and increases the risk of NEC by promoting colonization with more resistant pathogens. To date no single causative bacterial agent has been identified; however, a generalized microbial imbalance of the intestinal flora with Firmicutes and Proteobacteria dominant dysbiotic patterns has been detected before the development of early or late onset NEC respectively [8, 9]. Even though in most cases of NEC no specific microorganism can be considered causative (all cultures were negative in the case of our patient), viruses and bacteria have been consistently implicated somewhere along the pathogenic process. For this reason, broad spectrum antibiotic therapy continues to be a mainstay of NEC treatment.

The role of probiotics use in the neonatal population has been highly controversial. To date, one of the largest studies assessing the effects of Lactobacillus reuteri versus controls showed no difference in mortality, nosocomial infection or NEC in the probiotic-treated and control infants [3]. However, additional safety and efficacy studies should be conducted before introducing a certain strain as standard practice in neonatal care.

Despite being vigorous, the infant had endured a difficult delivery, which took place after a three-day induction and a prolonged expulsive period. These hypoxic perinatal events may have contributed to the development of the disease. Although no cord gas was obtained, we know that the infant's peripheral blood supply was compromised at some point due to the high lactate levels and the increased base deficit (metabolic academia) seen in the first blood gas at 10 hours of life. Another important contributing factor was the PDA, which was revealed in the first Echo at 24 hours of life and persisted open with significant left-to-right shunt until his seventh day of life. Heart disease has been frequently associated with an increased risk for NEC in term infants $[5,6,10]$. This is secondary to poor/decreased blood flow to the mesenteric vessels (left-to-right shunt), which produces hypoxic-ischemic failure.

Other described risk factors for NEC in term neonates include umbilical artery catheterization (UAC). Some studies with Doppler sonography reveal decreased mesenteric blood flow in neonates with umbilical artery catheters, even if they are properly placed [6]. UAC, along with narcotic use, congenital heart disease and perinatal hypoxic events, is also considered a risk factor for mortality from NEC in term infants [6].

Once the infant was diagnosed with NEC, elective medical treatment consists of broadspectrum antibiotics, such as AmpicillinGentamycin, with anaerobic antimicrobial therapy (Metronidazole). There is some controversy around the most effective first-line antibiotic therapy, and whether Metronidazole coverage should be added. Recent studies assessed the effectiveness of broad spectrum antibiotics in conjunction with Metronidazole to prevent the deterioration of NEC from stage II to III in full term and near term infants. Studies show that infants with stage II NEC are more likely to progress onto stage III if they are short on colostrum feeding, require fresh frozen plasma or cryoprecipitate transfusions or have an elevated C-reactive protein [2, 4]. Additionally, they found that the rate of deterioration from stage II NEC to stage III NEC did not vary significantly between the group of infants who were treated with Metronidazole and the group of those who were not, and that the mortality rates in both groups was similar [2]. Therefore, these studies suggest that broad spectrum antibiotics administered along with Metronidazole may not prevent deterioration from stage II to stage III in full-term and near term infants. Further research is needed to determine the optimal antibiotic therapy in infants with NEC.

Interestingly, a recent study showed a significant increase in intestinal fatty acidbinding protein (I-FABP) levels in full term infants with stage I NEC, as well as a correlation with the disease's severity [11]. The study suggests that serum I-FABP can be used as an early biomarker for the diagnosis of NEC in term neonates. Moreover, monitoring the presence of highly sensitive 
and specific proinflammatory factors such as PAF, IL1, TNF, IL10 in the patients' blood can be a useful tool for early detection of NEC [12].

\section{Conclusion}

NEC is a rare condition among full term infants, and it is primarily associated with prematurity and low birth weight. In term infants, the pathogenic mechanism results from a conjunction of events that lay the foundation for the development of the disease. The infant described in this case report had multiple risk factors (perinatal hypoxia, dysbiosis, formula feeding, maternal narcotic use and PDA), which led to the onset of this devastating condition. Since NEC develops in a fulminant manner, the key lies in the ability to predict which infants are at high risk, which

\section{References}

1. Sharma R, Hudak ML. A clinical perspective of necrotizing enterocolitis: past, present, and future. Clin Perinatol 2013; 40(1):27-51.

2. Luo LJ, Li X, Yang KD, et al. Broad-spectrum antibiotic plus metronidazole may not prevent the deterioration of necrotizing enterocolitis from stage II to III in full-term and near-term infants: a propensity score-matched cohort study. Medicine 2015; 94(42):e1862.

3. Neu J. Probiotics and necrotizing enterocolitis. Clin Perinatol 2014; 41(4):967-978.

4. Ng PC, Chan KY, Poon TC. Biomarkers for prediction and diagnosis of necrotizing enterocolitis. Clin Perinatol 2013; 40(1):149159.

5. Marseglia L, Manti S, D'Angelo G, et al. Colonic stenosis post necrotizig enterocolitis in term newborn with acquired Cytomegalovirus infection. Chirurgia 2015; 110(2):175-178.

6. Short S, Papillon S, Berel D, et al. Late onset of necrotizing enterocolitis in the full term infant is associated with increased mortality: results from a two-center analysis. J Pediatr Surg 2015; 49(6):950-953. gives the Neonatology team the opportunity to anticipate and act in consequence. Prevention seems to be the most effective strategy, given that once the disease becomes clinically evident it means that a systemic inflammatory cascade has already been activated, and will likely result in multiorgan injury.

\section{Consent}

Written informed consent was obtained from the patient's parents for publication of this case report and accompanying images. A copy of the consent is available for review by the Editor-in-Chief of this journal.

\section{Conflict of interest}

The author(s) declare that they have no competing interests.

7. Burjonrappa SC, Schwartzberg D. Chromosomal microarray testing in NEC: a case report. J Neonatal Surg 2016; 5(3):34.

8. Gritz EC, Bhandari V. The human neonatal gut microbiome: a brief review. Front Pediatr 2015; 3:17.

9. Elgin TG, Kern SL, McElroy SJ. Development of the neonatal intestinal microbiome and its association with necrotizing enterocolitis. Clin Ther 2016; 38(4):706-715.

10. Christensen RD1, Lambert DK, Baer VL, Gordon. Necrotizing Enterocolitis in Term Infants. Clin Perinatol 2013; 40(1):69-78.

11. Huang-Fu CR, Li $P$, Tian YF. Clinical significance of serum intestinal fatty acidbinding protein in full term infants with necrotizing enterocolitis. Zhongguo Dang Dai Er Ke Za Zhi 2014; 16 (11):1125-1128.

12. Wang W, Xue L, Ma T, Li Y, Li Z. Nonintervention observation: Dynamic evolution laws of inflammatory response in necrotizing enterocolitis. Exp Ther Med 2016; 12(3):17701774. 